\title{
Self-Calibrating Imaging Polarimetry
}

\author{
Yoav Y. Schechner \\ Dept. Electrical Engineering \\ Technion - Israel Institute of Technology \\ Haifa 32000, Israel \\ yoaveee.technion.ac.il \\ http://www.ee.technion.ac.il/ yoav
}

\begin{abstract}
To map the polarization state (Stokes vector) of objects in a scene, images are typically acquired using a polarization filter (analyzer), set at different orientations. Usually these orientations are assumed to be all known. Often, however, the angles are unknown: most photographers manually rotate the filter in coarse undocumented angles. Deviations in motorized stages or remote-sensing equipment are caused by device drift and environmental changes. This work keeps the simplicity of uncontrolled uncalibrated photography, and still extracts from the photographs accurate polarimetry. This is achieved despite unknown analyzer angles and the objects' Stokes vectors. The paper derives modest conditions on the data size, to make this task wellposed and even over-constrained. The paper then proposes an estimation algorithm, and tests it in real experiments. The algorithm demonstrates high accuracy, speed, simplicity and robustness to strong noise and other signal disruptions.
\end{abstract}

\section{Introduction}

Polarization is used in various domains of computational photography, as displays [1, 25, 31], rendering [3] and imaging $[2,3,5,11,16,17,32,34,42,45]$. In imaging, polarization picture post-processing $\left(P^{4}\right)$ is used for solving inverse problems [36], involving scattering [13, 22, 23, $36,40,43]$, surface reflection $[6,9,12,14,18,24,30,36$, $38,46,47]$ and remote sensing [7, 15, 21, 37, 44]. Biological vision uses $P^{4}$ for navigation, communication and predation.

It has often been thought that quantifying polarization in imaging and vision requires well aligned polarizing filters (analyzers). Imaging using a known set of analyzer angles is the foundation of state-of-the-art imaging polarimetry and many of its applications. This, however, is con-

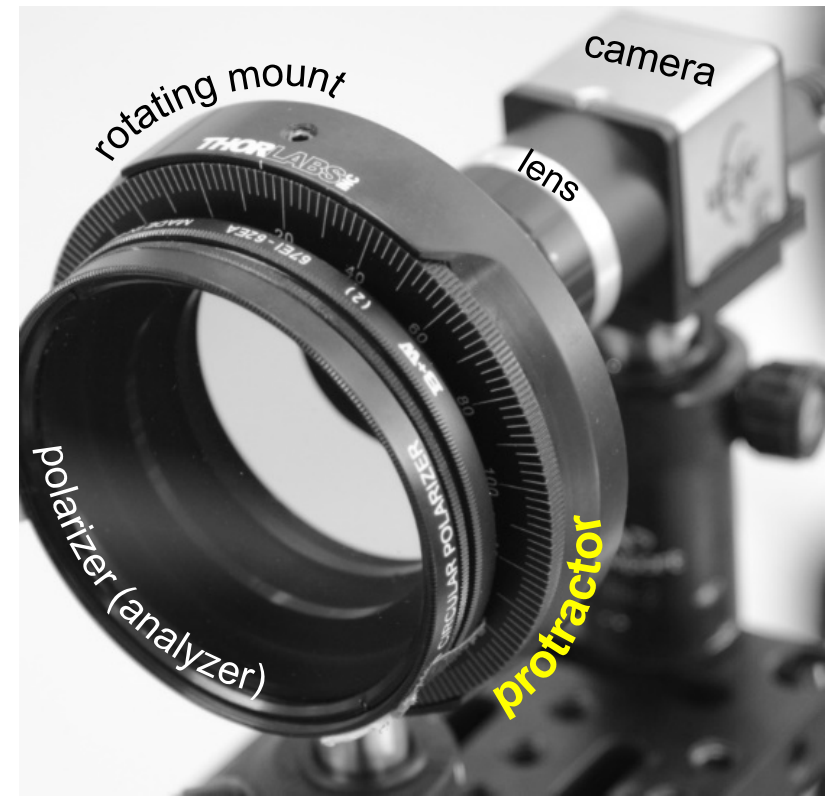

Figure 1. Polarimetric imaging has typically required a polarizing filter (analyzer) set to known angles. Commonly, the filter rotates using a controlled closed-loop motor, or manually with a fine protractor. This work seeks to dismiss the fine protractor or control, and still enable polarimetric imaging. Thus, the simplicity of uncontrolled amateur photography can be tamed for scientific measurements.

trary to the easy unconstrained practice of photographers. In common photography, a free-rolling polarizer is mounted on the camera lens, rotating coarsely by the wish of the photographer, without documented angles. This work retains this ease of use, while achieving accurate polarimetry of imaged objects, on a par with controlled measurements. Specifically, it is desirable to do photography as with the system in Fig. 1, but dismiss the protractor. Essentially, this means self-calibration, based on the unknown observed scene. Even scientific-grade instruments need to counter 
system drift and errors. Hence, remote sensing [7, 28] and astronomic polarimetric imagers $[21,41]$ require elaborate on-board calibrations. Potentially, scene-based selfcalibration can simplify or improve some construction and operation aspects of such imagers.

This paper presents computational self-calibration of a polarimetric imager. No additional optical hardware is required. No formulation of statistical scene priors (in space and polarization) is incorporated. No assumption is made regarding source separation. Rather, the paper formulates the modest data size requirements, to make the problem well-posed or over constrained. A proposed algorithm involves simple steps: a rough set of initial analyzer angles is assumed, followed by linear least-squares (LS) regressions. Each regression is non-iterative, since the data size required is small. We conducted a couple of quick experiments in uncontrolled environments, and the algorithm demonstrated success.

\section{Model}

In this paper, instrument polarizance is not part of the unknowns to be determined. Without essential loss of generality, suppose unit-polarizance of the analyzer. This polarizance is nearly met even by photography-grade filters. The paper deals with linear polarization, which is by far more dominant than circular polarization in the natural world. The paper does not deal with radiometric (unpolarized) calibration. Hence, the global unpolarized attenuation due to the filter, lens and detector array is not part of the variables: unpolarized transmissivity is assumed to be unit, without loss of generality.

\subsection{A Single Pixel}

Object point $o$ has an unpolarized radiance component $c_{o}$, and degree of linear polarization (DOLP) $p_{o}$. The angle of the polarization axis (phase angle) is $\theta_{o}$, relative to a global coordinate system. The object parameter set $\left\{c_{o}, p_{o}, \theta_{o}\right\}$ is unknown. At measurement (frame) $m$, the polarizing filter (analyzer) has an angle $\alpha_{m}$, relative to the same global coordinate system.

The acquired intensity is

$$
I_{m, o}=\left[c_{o}+c_{o} p_{o} \cos 2\left(\alpha_{m}-\theta_{o}\right)\right] / 2,
$$

which can also be expressed as

$$
I_{m, o}=\frac{1}{2}\left[\begin{array}{lll}
1 & \cos 2 \alpha_{m} & \sin 2 \alpha_{m}
\end{array}\right]\left[\begin{array}{c}
c_{o} \\
a_{o}^{\mathrm{c}} \\
a_{o}^{\mathrm{s}}
\end{array}\right],
$$

where

$$
a_{o}^{\mathrm{c}}=c_{o} p_{o} \cos 2 \theta_{o}, \quad a_{o}^{\mathrm{s}}=c_{o} p_{o} \sin 2 \theta_{o} .
$$

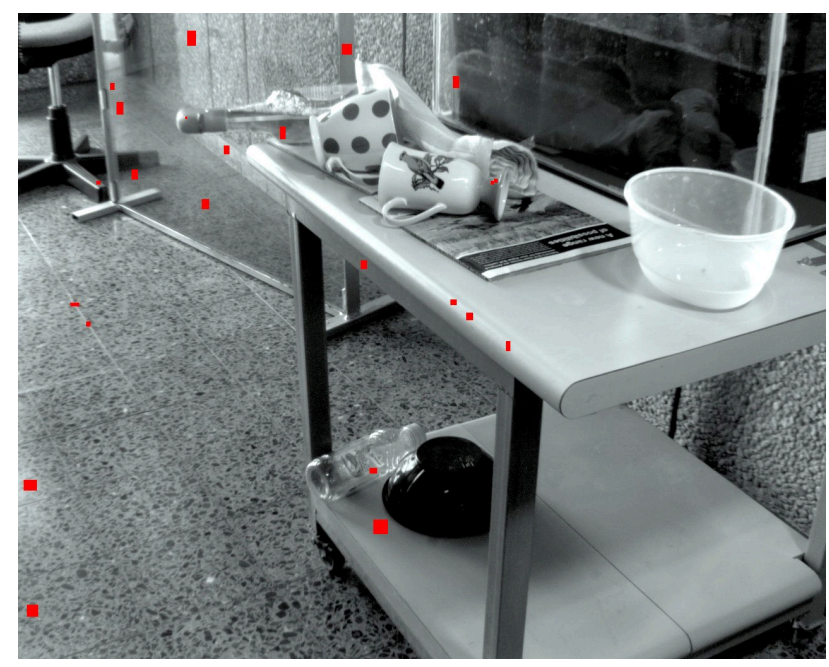

Figure 2. The corridor scene. Red rectangles show sampled object locations, from which data of polarized radiance readings was extracted.

While the unknown object is fixed, $N_{\mathrm{m}}$ measurements (frames) are acquired. In frame $m$, the polarizer is oriented at angle $\alpha_{m}$. There is diversity within the angle set. Thus, pixel $o$ yields

$\left[\begin{array}{c}I_{1, o} \\ I_{2, o} \\ \vdots \\ I_{m, o} \\ \vdots \\ I_{N_{\mathrm{m}}, o}\end{array}\right]=\frac{1}{2}\left[\begin{array}{lll}1 & \cos 2 \alpha_{1} & \sin 2 \alpha_{1} \\ 1 & \cos 2 \alpha_{2} & \sin 2 \alpha_{2} \\ \vdots & \vdots & \vdots \\ 1 & \cos 2 \alpha_{m} & \sin 2 \alpha_{m} \\ \vdots & \vdots & \vdots \\ 1 & \cos 2 \alpha_{N_{\mathrm{m}}} & \sin 2 \alpha_{N_{\mathrm{m}}}\end{array}\right]\left[\begin{array}{c}c_{o} \\ a_{o}^{\mathrm{c}} \\ a_{o}^{\mathrm{s}}\end{array}\right]$.

We may use the constraint $\sqrt{\left(a_{o}^{\mathrm{c}}\right)^{2}+\left(a_{o}^{\mathrm{s}}\right)^{2}} \leq c_{0}$.

\subsection{Multiple Pixels, Multiple Frames}

Assume that $\alpha_{m}$ is approximately space-invariant, or that spatial variations are largely predictable and compensated for. The field of view has many pixels. A set of $N_{\mathrm{o}}$ object regions is measured in all the frames, as shown in Fig. 2. The acquired data can expressed as

$$
\mathbf{D}=2\left[\begin{array}{cccccc}
I_{1,1} & I_{1,2} & . . & I_{1, o} & . . & I_{1, N_{\mathrm{o}}} \\
I_{2,1} & I_{2,2} & . . & I_{2, o} & . . & I_{2, N_{\mathrm{o}}} \\
\vdots & & & \vdots & & \vdots \\
I_{m, 1} & I_{m, 2} & . . & I_{m, o} & . . & I_{m, N_{\circ}} \\
\vdots & & & \vdots & & \vdots \\
I_{N_{\mathrm{m}}, 1} & I_{N_{\mathrm{m}}, 2} & . . & I_{N_{\mathrm{m}}, o} & . . & I_{N_{\mathrm{m}}, N_{\mathrm{o}}}
\end{array}\right] .
$$

Generalizing Eq. (4) for Eq. (5) yields

$$
\mathbf{D}=\mathbf{P O},
$$


where

$$
\mathbf{P}=\left[\begin{array}{ccc}
1 & \cos 2 \alpha_{1} & \sin 2 \alpha_{1} \\
1 & \cos 2 \alpha_{2} & \sin 2 \alpha_{2} \\
\vdots & \vdots & \vdots \\
1 & \cos 2 \alpha_{m} & \sin 2 \alpha_{m} \\
\vdots & \vdots & \vdots \\
1 & \cos 2 \alpha_{N_{\mathrm{m}}} & \sin 2 \alpha_{N_{\mathrm{m}}}
\end{array}\right]
$$

and

$$
\mathbf{O}=\left[\begin{array}{cccccc}
c_{1} & c_{2} & . . & c_{o} & . . & c_{N_{\circ}} \\
a_{1}^{\mathrm{c}} & a_{2}^{\mathrm{c}} & . . & a_{o}^{\mathrm{c}} & . . & a_{N_{\mathrm{o}}}^{\mathrm{c}} \\
a_{1}^{\mathrm{s}} & a_{2}^{\mathrm{s}} & . . & a_{o}^{\mathrm{s}} & . . & a_{N_{\mathrm{o}}}^{\mathrm{s}}
\end{array}\right] .
$$

\subsection{Controlled Imaging Polarimetry}

Controlled imaging polarimetry has access to a groundtruth set of analyzer angles $\left\{\alpha_{m}^{\text {true }}\right\}_{m=1}^{N_{\mathrm{m}}}$, up to the protractor measurement error. Through Eq. (7), this set is equivalent to the true analyzer matrix, denoted $\mathbf{P}_{\text {true }}$. This matrix is used to perform rather standard polarimetry, e.g., using LS fitting (see Sec. 4.1). The result is the set of object Stokes vectors, organized in a matrix denoted $\mathbf{O}_{\text {measured. }}$. The mean-squared-error (MSE) between the model and the data is equivalent to

$$
f_{\text {true }}=\left\|\mathbf{D}-\mathbf{P}_{\text {true }} \mathbf{O}_{\text {measured }}\right\|^{2} .
$$

The value of $f_{\text {true }}$ is not zero, due to noise and other disturbances in $\mathbf{D}$.

In the $o^{\text {th }}$ column of $\mathbf{O}_{\text {measured }}$, the second and third elements form the vector $\mathbf{a}_{o}^{\text {measured }}$, while the first element is $c_{o}^{\text {measured }}$. The measured (denoted true) object DOLP and phase angle are respectively

$$
p_{o}^{\text {true }}=\frac{\left\|\mathbf{a}_{o}^{\text {measured }}\right\|_{2}}{c_{o}^{\text {measured }}} \quad \theta_{o}^{\text {true }}=\frac{\angle \mathbf{a}_{o}^{\text {measured }}}{2} .
$$

\section{Task}

Suppose the analyzer rotates uncontrolled, and there is no protractor to measure its state. The task is to estimate the analyzer set of orientations $\left\{\alpha_{m}\right\}$ and the polarization parameters of all object points $\left\{c_{o}, p_{o}, \theta_{o}\right\}_{o}^{N_{\mathrm{o}}}$. There is a fundamental ambiguity. As seen in Eq. (1), the measurements are unaffected by the absolute value of either $\alpha_{m}$ or $\theta_{o}$, but by the difference $\left(\alpha_{m}-\theta_{o}\right)$. Suppose all of the values of the unknown sets $\left\{\alpha_{m}\right\}_{m=1}^{N_{\mathrm{m}}}$ and $\left\{\theta_{o}\right\}_{o=1}^{N_{\mathrm{o}}}$ are shifted by an arbitrary global bias $\beta$, i.e.,

$$
\alpha_{m} \mapsto \alpha_{m}+\beta, \quad \theta_{o} \mapsto \theta_{o}+\beta, \quad \forall m, \forall o .
$$

Then, any measured value (Eq. 1) is unaffected by this bias. Since the measurements are unaffected by the bias, then this bias cannot be detected. Hence, all the recovered angles suffer from a global shift ambiguity.
Without loss of generality, set

$$
\alpha_{1}=0 .
$$

This sets an origin of a coordinate system, to which all other angles refer. Consequently, all the recovered angles $\left\{\alpha_{m}\right\}_{m=2}^{N_{\mathrm{m}}}$ and $\left\{\theta_{o}\right\}_{o=1}^{N_{\mathrm{o}}}$ are recovered relative to the first analyzer angle. ${ }^{1}$ Then,

$$
\mathbf{P}=\left[\begin{array}{ccc}
1 & 1 & 0 \\
1 & \cos 2 \alpha_{2} & \sin 2 \alpha_{2} \\
\vdots & \vdots & \vdots \\
1 & \cos 2 \alpha_{m} & \sin 2 \alpha_{m} \\
\vdots & \vdots & \vdots \\
1 & \cos 2 \alpha_{N_{\mathrm{m}}} & \sin 2 \alpha_{N_{\mathrm{m}}}
\end{array}\right] .
$$

From Eq. (5), the left hand side of Eq. (6) has $N_{\mathrm{m}} N_{\mathrm{o}}$ measurements. From Eqs. $(8,13)$, the number of unknowns on the right hand side of Eq. (6) is $\left[\left(N_{\mathrm{m}}-1\right)+3 N_{\mathrm{o}}\right]$. To sufficiently constrain the task, a necessary condition is that

$$
N_{\mathrm{m}} N_{\mathrm{o}} \geq\left(N_{\mathrm{m}}-1\right)+3 N_{\mathrm{o}} .
$$

Rearranging terms, Eq. (14) is satisfied in the following setups:

- $N_{\mathrm{o}} \geq 3$ while $N_{\mathrm{m}} \geq 4$

- $N_{\mathrm{o}}=2$ while $N_{\mathrm{m}} \geq 5$.

Generally, images contain much more than two or three regions having different polarizations. Hence, Eq. (14) is generally satisfied in practice.

\section{Self Calibration by Optimization}

Assuming the data is sufficient, the self calibration task is accomplished by optimization. As cost function, consider

$$
f=\|\mathbf{D}-\mathbf{P O}\|^{2},
$$

similarly to Eq. (9). The unknown parameters of $f$ include the set of angles of the polarizer $\Phi^{\mathrm{p}}=\left\{\alpha_{m}\right\}_{m=2}^{N_{\mathrm{m}}}$. In addition, the unknown parameters of $f$ include the set of object characteristics, i.e. $\mathbf{O}$. Then, we seek

$$
\left\{\widehat{\Phi}^{\mathrm{p}}, \widehat{\mathbf{O}}\right\}=\arg \min _{\Phi^{\mathrm{p}}, \mathbf{O}} f, \text { s.t. } \mathbf{O} \in \mathcal{C} .
$$

Here $\mathcal{C}$ is a constraint (convex cone) on the feasible object Stokes vectors:

$$
\mathcal{C}=\bigcap_{o=1}^{N_{\mathrm{o}}}\left(0 \leq c_{o}\right) \cap\left(\left\|\left[a_{o}^{\mathrm{c}}, a_{o}^{\mathrm{s}}\right]\right\|_{2} \leq c_{o}\right) .
$$

Assuming moderately polarized objects, the chance is small for constraint $\mathcal{C}$ to be active. To ease the optimization, we unconstrain the problem, i.e. solve Eq. (16) ignoring $\mathcal{C}$.

\footnotetext{
${ }^{1}$ The global shift parameter can be resolved by a simple prior in postprocessing. To align the global origin with gravity, one may set the origin according to the phase angle at an object $o$ known to be horizontal, such as a water surface.
} 


\subsection{Given $P$}

Denote by vector $\hat{\mathbf{o}}$ the column-stack of $\widehat{\mathbf{O}}$. Let $\mathbf{d}$ be the column-stack of $\mathbf{D}$. Let $\mathbf{0}_{\mathrm{p}}$ denote a matrix of zeros, of the dimensions of $\mathbf{P}$. Suppose $\Phi^{\mathrm{p}}$ (and thus $\mathbf{P}$ ) is given. Then, an unconstrained LS estimate $\hat{o}$ is

$$
\hat{\mathbf{o}}=\left[\mathbf{M}_{\mathrm{p}}^{t} \mathbf{M}_{\mathrm{p}}\right]^{-1} \mathbf{M}_{\mathrm{p}}^{t} \mathbf{d},
$$

where $t$ denotes transposition and

$$
\mathbf{M}_{\mathrm{p}}=\left[\begin{array}{lllll}
\mathbf{P} & \mathbf{0}_{\mathrm{p}} & \mathbf{0}_{\mathrm{p}} & \ldots & \mathbf{0}_{\mathrm{p}} \\
\mathbf{0}_{\mathrm{p}} & \mathbf{P} & \mathbf{0}_{\mathrm{p}} & \ldots & \mathbf{0}_{\mathrm{p}} \\
\mathbf{0}_{\mathrm{p}} & \mathbf{0}_{\mathrm{p}} & \mathbf{P} & \ldots & \mathbf{0}_{\mathrm{p}} \\
\vdots & \vdots & & & \vdots \\
\mathbf{0}_{\mathrm{p}} & \mathbf{0}_{\mathrm{p}} & \mathbf{0}_{\mathrm{p}} & \ldots & \mathbf{P}
\end{array}\right]
$$

\subsection{Given $O$}

Suppose $\mathbf{O}$ is given. Define

$$
\widetilde{\mathbf{O}}=\left[\begin{array}{cccccc}
a_{1}^{\mathrm{c}} & a_{2}^{\mathrm{c}} & . . & a_{o}^{\mathrm{c}} & . . & a_{N_{\mathrm{o}}}^{\mathrm{c}} \\
a_{1}^{\mathrm{s}} & a_{2}^{\mathrm{s}} & . . & a_{o}^{\mathrm{s}} & . . & a_{N_{\mathrm{o}}}^{\mathrm{s}}
\end{array}\right]
$$

Let 1 be a column vector of length $N_{\mathrm{m}}$, all of whose elements are 1's. Define

$$
\widetilde{\mathbf{D}} \equiv \mathbf{D}-\mathbf{1}\left[c_{1} c_{2} \ldots c_{N_{\mathrm{o}}}\right] .
$$

Then, from Eqs. $(6,8,13,20,21)$,

$$
\widetilde{\mathbf{D}}=\left[\begin{array}{cc}
1 & 0 \\
\cos 2 \alpha_{2} & \sin 2 \alpha_{2} \\
\cos 2 \alpha_{3} & \sin 2 \alpha_{3} \\
\vdots & \vdots \\
\cos 2 \alpha_{N_{\mathrm{m}}} & \sin 2 \alpha_{N_{\mathrm{m}}}
\end{array}\right] \widetilde{\mathbf{O}}
$$

Hence,

$$
\widetilde{\mathbf{D}}^{t}=\widetilde{\mathbf{O}}^{t}\left[\begin{array}{cc}
1 & \widetilde{\mathbf{P}} \\
0 &
\end{array}\right]
$$

where

$$
\widetilde{\mathbf{P}}=\left[\begin{array}{ccc}
\cos 2 \alpha_{2} & \cos 2 \alpha_{3} \ldots & \cos 2 \alpha_{N_{\mathrm{m}}} \\
\sin 2 \alpha_{2} & \sin 2 \alpha_{3} \ldots & \sin 2 \alpha_{N_{\mathrm{m}}}
\end{array}\right]
$$

is unknown.

Let $\tilde{\mathbf{d}}$ be the column-stack of $\widetilde{\mathbf{D}}^{t}$. The first $N_{\mathrm{o}}$ elements of $\tilde{\mathbf{d}}$ do not depend on the unknown $\widetilde{\mathbf{P}}$, and thus can be cropped. Let $\tilde{\mathbf{d}}_{\mathrm{r}}$ be a cropped version $\tilde{\mathbf{d}}$, in which the first $N_{\text {o }}$ elements of $\tilde{\mathbf{d}}$ are excluded. Denote by vector $\mathbf{p}$ the column-stack of $\widetilde{\mathbf{P}}$. An unconstrained LS estimate of $\mathbf{p}$ is

$$
\hat{\mathbf{p}}=\left[\mathbf{M}_{\mathrm{o}}^{t} \mathbf{M}_{\mathrm{o}}\right]^{-1} \mathbf{M}_{\mathrm{o}}^{t} \tilde{\mathbf{d}}_{\mathrm{r}},
$$

where

$$
\mathbf{M}_{\mathrm{o}}=\left[\begin{array}{lllll}
\widetilde{\mathbf{O}}^{t} & \mathbf{0}_{\mathrm{o}} & \mathbf{0}_{\mathrm{o}} & \ldots & \mathbf{0}_{\mathrm{o}} \\
\mathbf{0}_{\mathrm{o}} & \widetilde{\mathbf{O}}^{t} & \mathbf{0}_{\circ} & \ldots & \mathbf{0}_{\circ} \\
\mathbf{0}_{\mathrm{o}} & \mathbf{0}_{\mathrm{o}} & \widetilde{\mathbf{O}}^{t} & \ldots & \mathbf{0}_{\circ} \\
\vdots & \vdots & & & \vdots \\
\mathbf{0}_{\mathrm{o}} & \mathbf{0}_{\mathrm{o}} & \mathbf{0}_{\mathrm{o}} & \ldots & \widetilde{\mathbf{O}}^{t}
\end{array}\right]
$$

Here $\mathbf{0}_{\mathrm{o}}$ denotes a matrix of zeros, of the dimensions of $\widetilde{\mathbf{O}}^{t}$.

The resulting vector is equivalent to matrix $\widehat{\mathbf{P}}$, the estimate of Eq. (24). Let $\mathbf{v}_{m+1}$ be the $m^{\prime}$ th column of $\widehat{\mathbf{P}}$. Based on the structure of Eq. (24), the analyzer orientation is estimated by

$$
\hat{\alpha}_{m+1}=(1 / 2) \angle \mathbf{v}_{m+1}, \quad m=1 \ldots\left(N_{\mathrm{m}}-1\right) .
$$

\subsection{Iterative Algorithm: Alternating Minimization}

Neither $\mathbf{P}$ nor $\mathbf{O}$ are known. A practical approach to solution is alternating minimization: a sequence of iterations combining the steps of Secs. 4.1,4.2:

Step 1: Initialize the set of analyzer orientations $\Phi_{(0)}^{\mathrm{p}}$ using rough assessments.

Per iteration $k=1,2,3, \ldots$

Step 2: Based on $\Phi_{(k-1)}^{\mathrm{p}}$, estimate $\widehat{\mathbf{O}}_{(k)}$ using Eqs. $(5,13,18,19)$.

Step 3: Based on $\widehat{\mathbf{O}}_{(k)}$, estimate the orientation set $\widehat{\Phi}_{(k)}^{\mathrm{p}}$, using Eqs. $(5,8,20-27)$.

Iterate steps 2 and 3 until convergence.

Define the normalized MSE cost,

$$
e=\frac{f}{f_{\text {true }}} .
$$

Sec. 5 details experiments. Here, a typical plot of $e(k)$ is shown in Fig. 3. Indeed, the cost function monotonically decreases with iterations. Recall that the aim is to seek performance on a par with standard polarimetry: $\widehat{\Phi}^{\mathrm{p}} \rightarrow \Phi_{\text {true }}^{\mathrm{p}}$ and $\widehat{\mathbf{O}} \rightarrow \mathbf{O}_{\text {measured. }}$ Consequently, one would ideally wish to obtain $e \rightarrow 1$, but not $e<1$. As Fig. 3 shows, the algorithm crosses the desired value $e=1$, and stagnates slightly below this value.

In the task of this paper, an algorithm has no access to $\Phi_{\text {true }}^{\mathrm{p}}$ and $e$. Hence, repeated iterations seek to minimize $f$, even if Eq. (28) yields $e<1$, slightly drifting downwards from $e=1$. This may happen as the algorithm seeks to accommodate noise in $\mathbf{D}$.

\section{Experiments}

We mounted a Fujinon HF9HA-1B lens on a uEye monochrome machine-vision camera, eliminating $\gamma$ correction. In each experiment, the camera gain was fixed. 


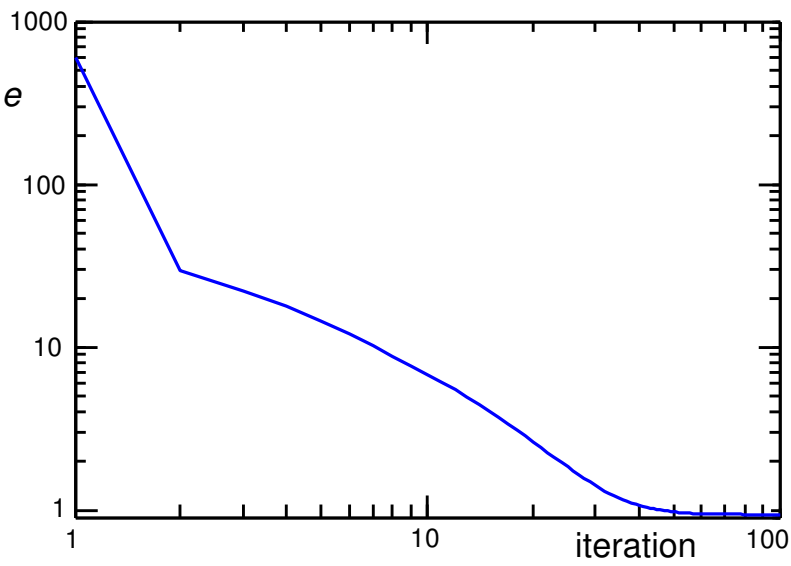

Figure 3. The optimization process, using data of the corridor scene (corresponding to Fig. 2). The normalized MSE criterion $e$ falls off with iterations. Within a few iterations, $e$ decreases by orders of magnitude towards the desired value $e=1$. Afterwards, $e$ decays very slowly.

The exposure time per frame was $200 \mathrm{~ms}$, averaging out regular fluorescent light flicker caused by $50 \mathrm{~Hz} \mathrm{AC}$ power. A standard photography circular polarizer $(\mathrm{B}+\mathrm{W} 67 \mathrm{~mm})$ was used as a linear polarization analyzer, by facing the filter to the scene properly. The filter was tied (by an adaptor ring) to a manual rotation mount (Thorlabs RSP2), that has a protractor. The protractor scale has $2^{o}$ increments. Using this mount, ground-truth analyzer angles were read with $\approx \pm 1^{\circ}$ precision. The system, shown in Fig. 1, was set on a tripod (Fig. 4) and covered by a black hood.

To obtain ground-truth analyzer angles in this setup, each angle was carefully set and read prior to frame capture. For this reason, it took overall several minutes to capture any set of polarization-filtered frames across all angles. In subsequent analysis, some small image regions were selected (mouse clicked): these regions appeared to have variations across frames (indicating measurable DOLP), avoiding saturated bright or dark areas. The regions were diverse, exhibiting intensity maxima in different frames.

\subsection{Corridor}

The first experiment was at the corridor scene, shown in Fig. 2. It was done during the day. Hence part of the illumination is from partly-clouded skylight, sunlight reflected off walls, and overhead indoor fluorescent bulbs. In Fig. 2, red rectangles show sampled object regions, from which data of polarized radiance readings were extracted. The scene had non-ideal conditions and un-modeled effects, as may occur in typical uncontrolled photography. Specifically, lighting coming from the window varied while data was captured. This affected objects differently, depending on their normal, distance from the window and shadowing configuration rel-

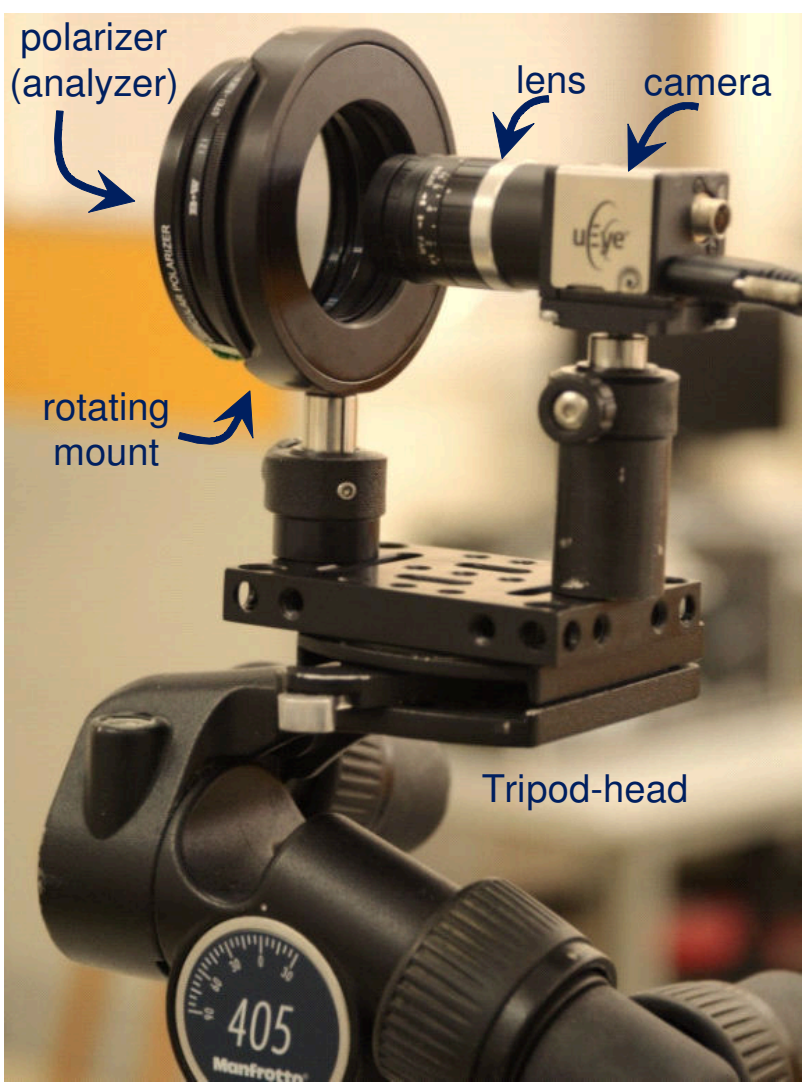

Figure 4. The experimental system is shown in Fig. 1. The rotating mount includes protractor scales in $2^{\circ}$ increments. The protractor provides ground-truth analyzer angles, for verification. The system was mounted on a tripod, and covered by a dark hood.

ative to the lighting components. The intensity readout $I$ is plotted vs. the true analyzer angles $\alpha_{m}^{\text {true }}$ in Fig. 5, for some regions. Some plots are well-behaved, while others show systematic un-modelled drifts over time.

In our task, $\alpha_{m}^{\text {true }}$ is unknown. Hence, plots as Fig. 5 presumably cannot be made in order to prune non-cooperative regions, prior to the self-calibration algorithm. For this reason, the algorithm was run on data whose source is from both kinds of regions. The algorithm appeared to hold despite these un-modelled un-cooperative real-world effects.

We used $N_{m}=5$ frames out of the data captured. To emulate rough prior knowledge, the initial angle-set $\Phi_{(0)}^{\mathrm{p}}$ was random, each angle deviating up to $\pm 30^{\circ}$ from the corresponding true value. Final results were insensitive to the random initialization, in repeated runs. However, convergence (as illustrated in Fig. 3) varied with initialization, taking between a handful to dozens of iterations. Typical results are shown in Table 1. Here $N_{\mathrm{o}}=21$. The results in Table 1 are within the measurement error of the true angles.

The polarimetric sensing of the object regions is also consistent. Using the self-calibrated analyzer angles $\left\{\hat{\alpha}_{m}\right\}_{m=1}^{5}$, the object matrix $\widehat{\mathbf{O}}$ is obtained by 

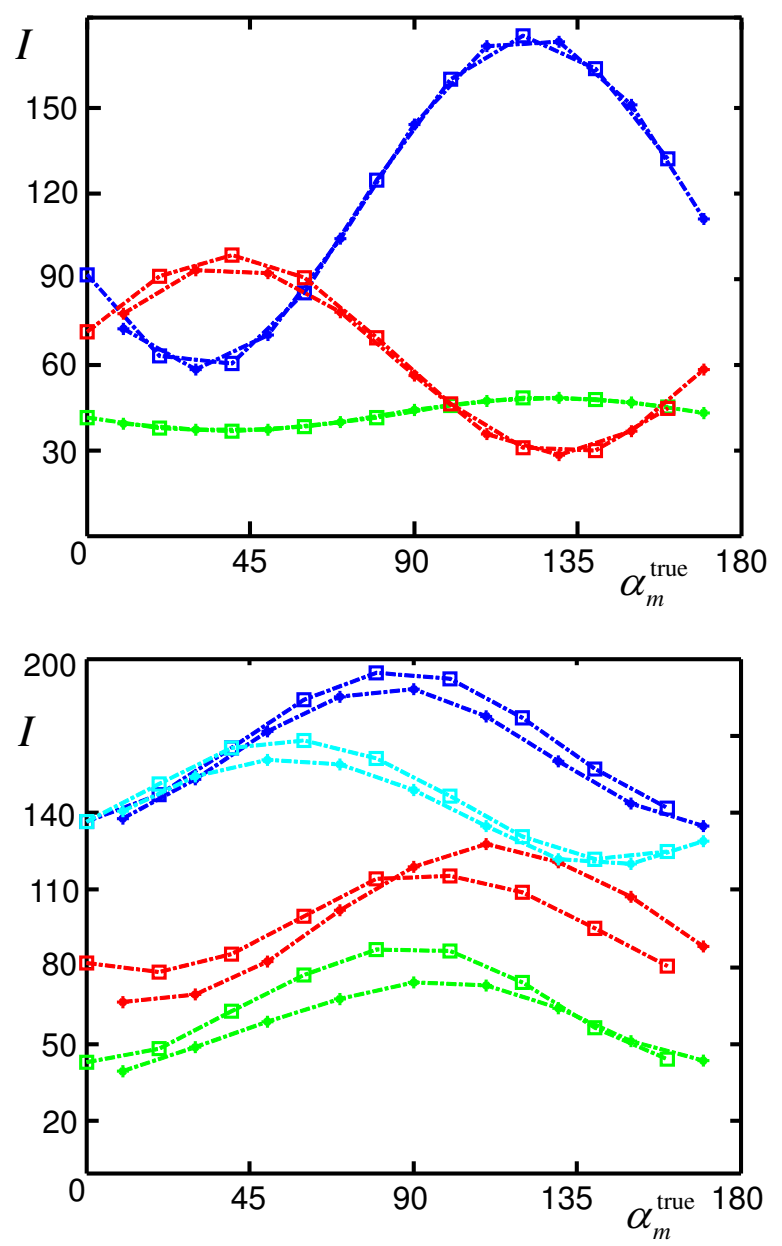

Figure 5. Intensity readout $I$ vs. true analyzer angles $\alpha_{m}^{\text {true }}$, for sample regions of those marked in Fig. 2. Per region, there are two adjacent plots corresponding to different session minutes. [Top] Well-behaved regions, in each of which the plot-pair is rather consistent. [Bottom] Non-cooperative regions, which have significant inconsistencies within each plot-pair, presumably due to changes of sky lighting by cloud motion.

\begin{tabular}{l|lllll}
\hline Set of angles & $\alpha_{1}$ & $\alpha_{2}$ & $\alpha_{3}$ & $\alpha_{4}$ & $\alpha_{5}$ \\
\hline \hline Initial $\Phi_{(0)}^{\mathrm{p}}$ & $0^{\circ}$ & $64^{\circ}$ & $58^{\circ}$ & $145^{\circ}$ & $148^{\circ}$ \\
100 iterations & $0^{o}$ & $39.5^{o}$ & $79.6^{o}$ & $119.1^{\circ}$ & $139.6^{\circ}$ \\
\hline True $\pm 1^{o}$ & $0^{o}$ & $40^{\circ}$ & $80^{\circ}$ & $120^{\circ}$ & $140^{\circ}$ \\
\hline
\end{tabular}

Table 1. Self-calibration results of the corridor experiment, with $N_{\mathrm{o}}=21$. Angle $\alpha_{1}$ is fixed, the others are variables. The random initial guesses are within $\pm 30^{\circ}$ of the true angles. Monotonicity is not enforced. After iterations, the results are consistent with the angular measurement errors.

Eqs. $(5,13,18,19)$. In the $o^{\text {th }}$ column of $\widehat{\mathbf{O}}$, the second and third elements form the vector $\hat{\mathbf{a}}_{o}$, while the first element is $\hat{c}_{o}$. The estimated object DOLP and phase angle are respec-
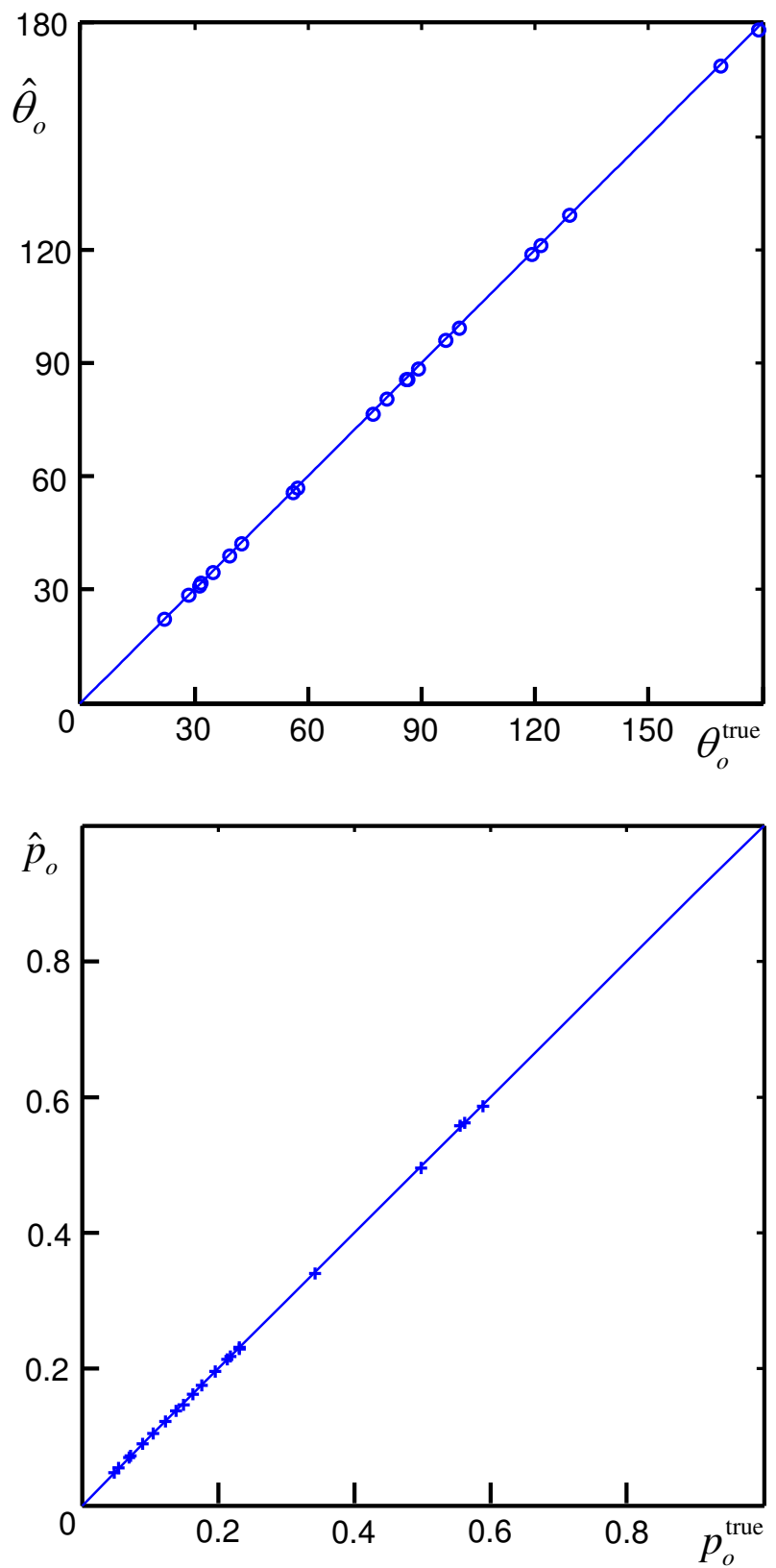

Figure 6. Resulting self-calibrated polarimetry in the corridor experiment. The estimated DOLP $\hat{p}_{o}$ and polarization (phase) angle $\hat{\theta}_{o}$ are in tight agreement with values measured using the known set $\left\{\alpha_{m}^{\text {true }}\right\}_{m=1}^{5}$.

tively

$$
\hat{p}_{o}=\frac{\left\|\hat{\mathbf{a}}_{o}\right\|_{2}}{\hat{c}_{o}} \quad \hat{\theta}_{o}=\frac{\angle \hat{\mathbf{a}}_{o}}{2} .
$$

Figure 6 compares these values to those measured (Eq. 10) using $\left\{\alpha_{m}^{\text {true }}\right\}_{m=1}^{5}$. 


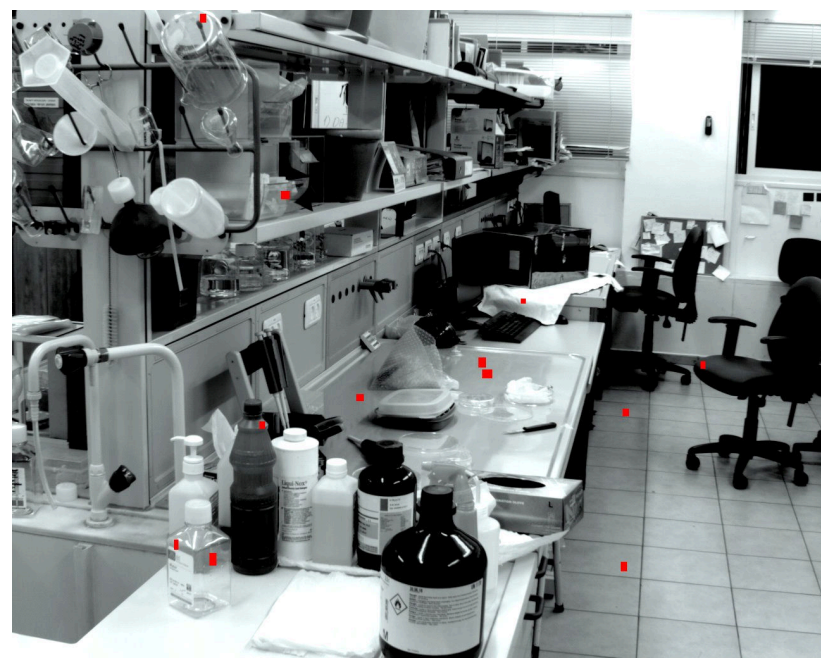

Figure 7. The $l a b$. Red rectangles show sampled object locations, from which data of polarized radiance readings was extracted.

\subsection{Lab}

The second experiment was at a lab scene, shown in Fig. 7. Lighting was solely by an array of ceiling fluorescent bulbs. Also here, conditions have caveats and un-modeled effects typical to uncontrolled photography: people work and move in the lab (out of the field of view), and some bulb may be erratic. The intensity readout $I$ is plotted vs. the true analyzer angles $\alpha_{m}^{\text {true }}$ in Fig. 8, for 12 regions marked by red rectangles in Fig. 7. Here the filter orientations were rather arbitrary, but the $0^{\circ}$ state was sampled several times during the session. In Fig. 8, these repeated measurements indicate highly noisy data. All 12 spatial regions were used. From a single continuous $180^{\circ}$ interval pass of the rotation mount, 5 frames were taken.

Again, the algorithms performed despite the disturbances. As with the corridor experiment, the initial angleset $\Phi_{(0)}^{\mathrm{p}}$ was random, each angle deviating up to $\pm 30^{\circ}$ from the corresponding true value. Final results were insensitive to the random initialization, in repeated runs. Results are shown in Table 2, and are consistent with the angular measurement errors.

The polarimetric sensing of the object regions are consistent also in this experiment. Using the self-calibrated analyzer angles $\left\{\hat{\alpha}_{m}\right\}_{m=1}^{5}$, the object parameters are estimated (Eq. 29). Figure 9 compares estimations to measurements (Eq. 10) made using $\left\{\alpha_{m}^{\text {true }}\right\}_{m=1}^{5}$.

Software code and data are available at the SelfCalibrating Imaging Polarimetry research web page: webee.technion.ac.il/ $\sim$ yoav/research/polar-self-calib.html

\section{Discussion}

Imaging polarimetry has inherent self-calibrating capacity. This capacity is in analogy to other domains of com-

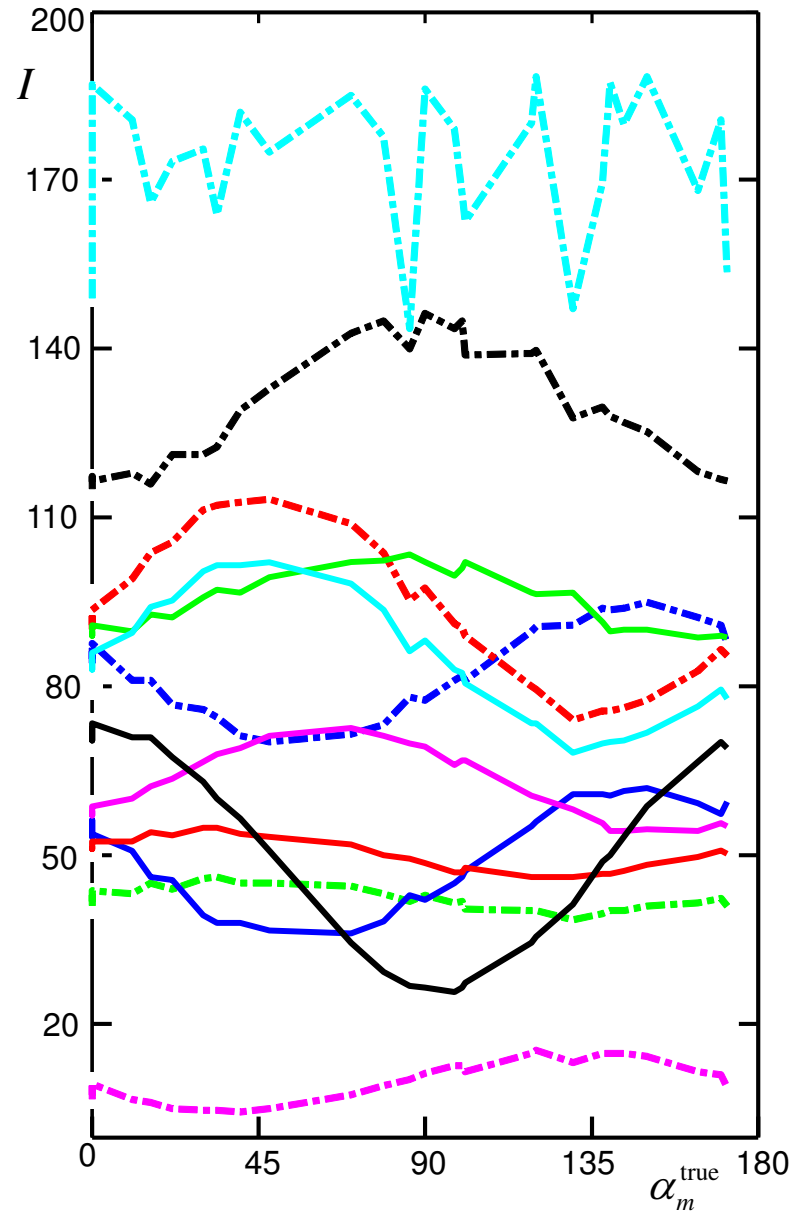

Figure 8. Intensity readout $I$ vs. true analyzer angles $\alpha_{m}$, for regions marked in Fig. 7. The entire orientation domain was repeatedly sampled during several minutes. Plots show significant inconsistencies relative to a smooth-cosine model.

\begin{tabular}{l|lllll}
\hline Set of angles & $\alpha_{1}$ & $\alpha_{2}$ & $\alpha_{3}$ & $\alpha_{4}$ & $\alpha_{5}$ \\
\hline \hline Initial $\Phi_{(0)}^{\mathrm{p}}$ & $0^{o}$ & $60^{\circ}$ & $68^{\circ}$ & $146^{\circ}$ & $116^{\circ}$ \\
100 iterations & $0^{o}$ & $48.6^{\circ}$ & $79.8^{\circ}$ & $120.0^{\circ}$ & $145.3^{\circ}$ \\
\hline True $\pm 1^{\circ}$ & $0^{o}$ & $48^{\circ}$ & $79^{\circ}$ & $119^{\circ}$ & $144^{\circ}$ \\
\hline
\end{tabular}

Table 2. Self-calibration results of the $l a b$ experiment, with $N_{\mathrm{o}}=12$. Angle $\alpha_{1}$ is fixed, the others are variables. The random initial guesses are within $\pm 30^{\circ}$ of the true angles. Monotonicity is not enforced. After iterations, the results are consistent with the angular measurement errors.

puter vision and computational photography (structure from motion [39], radiometric non-idealities [19, 27, 39], photometric stereo [33]). After setting some conditions on the data size, the paper proposes an estimation algorithm. The results imply several desirable merits: accuracy, speed, simplicity and handling of noise and other signal disruptions. Interestingly, attempting to solve Eq. (16) using brute ex- 

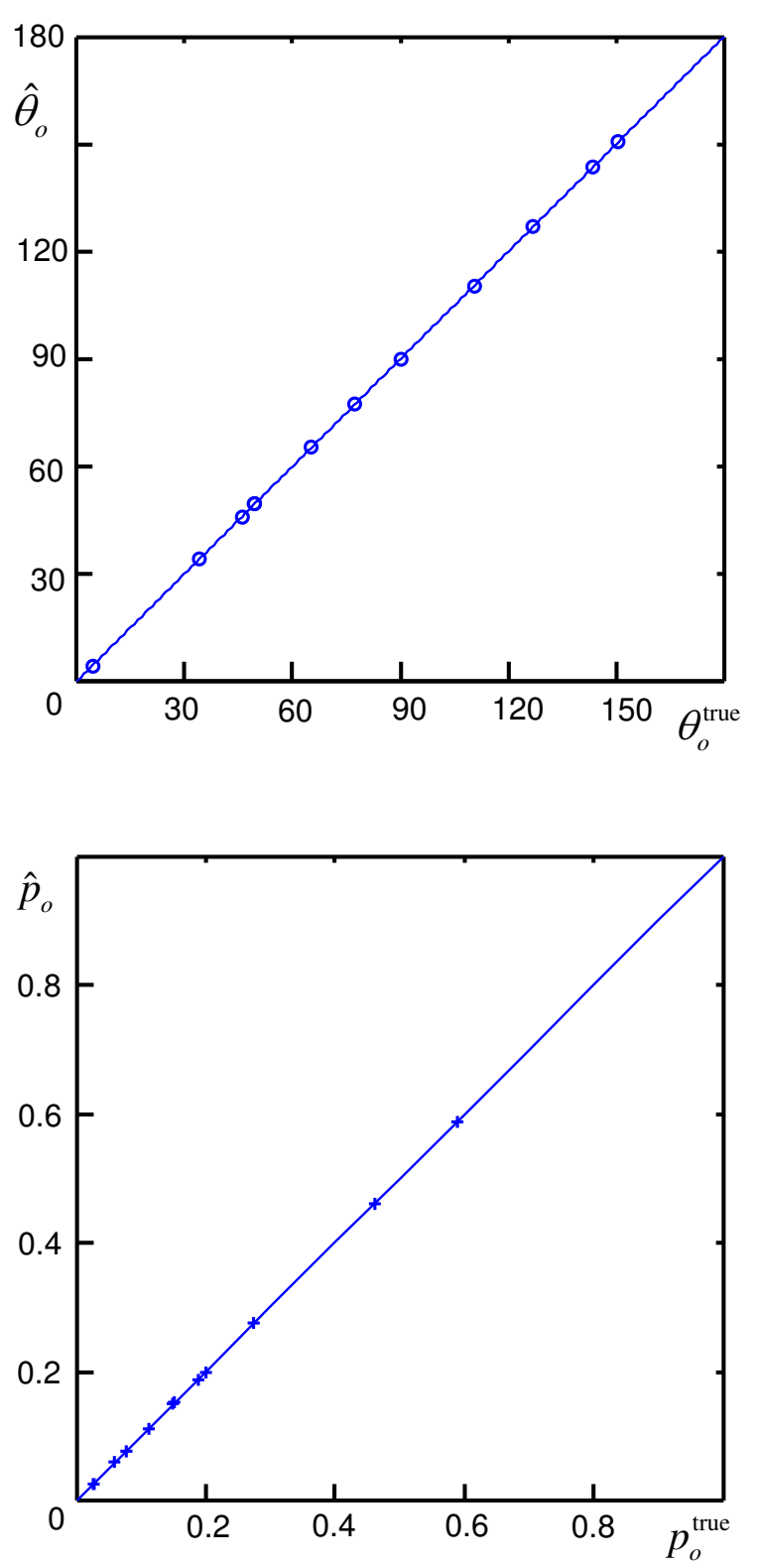

Figure 9. Self-calibrated polarimetry in the lab experiment. The estimated DOLP $\hat{p}_{o}$ and polarization (phase) angle $\hat{\theta}_{o}$ tightly agree with values measured using the known set $\left\{\alpha_{m}^{\text {true }}\right\}_{m=1}^{5}$.

haustive search often lead to wrong results in some of the angles, perhaps due to noise, while the LS regressions directly lead to the correct result. The paper has not proved theoretically the convergence of the method. Theoretical analysis may find failure domains. Nevertheless, the empirical results suggest that the problem has an inherent structure, that imposes projections to a correct solution. The theory needs to be further explored.

The breaking points of the problem and solutions are yet to be found and derived. How do accuracy and convergence depend on the noise in $\mathbf{D}$, be it sensor noise or region- dependent lighting variations? At some levels of disruption, the algorithm may break down: then robust estimators may need to be employed instead of, or in conjunction to LS fitting. A version of RANSAC or Hough Transform may then be developed for this task. No sophisticated priors on images and object polarization (e.g., independent component analysis, sparsity, smoothness) has so far been applied. Use of such priors should be helpful.

How is accuracy affected by the conditioning of $\mathbf{O}, \mathbf{P}$ and their singular values? Clearly, if all object regions have a very similar Stokes vector, or if all analyzer angles are very similar (close to 0 ), then the problem becomes illconditioned. Theoretical and numerical analysis is needed to clarify how performance degrades in near-degeneracy of partly polarized scenes or analyzer angle span. In practice, conditioning may not be a common problem: the user can easily rotate the filter sufficiently between frames, to ensure angular diversity, while scenes of interest often emanate a variety of phase angles across the field.

As all prior work on polarimetry, here the experimental image acquisition required mechanical stability, achieved by a common tripod. Nevertheless, for hand-held photographs, advances in computer vision now enable robust correspondence matchings and registration, despite unknown inter-frame camera motion. Hence, computer-vision can likely yield a sufficient set of corresponding measurements, to set $\mathbf{O}$ and $\mathbf{D}$.

There are several implications to this work. Photographers use standard cameras and free-rolling uncontrolled polarizing filters. They can be empowered to capture and quantify polarization of each pixel, without any change to the convenient photo gear. Then, they can employ polarization-based quantitative algorithms either for enhanced photography or scientific exploration. Second, selfcalibration can simplify scientific-grade polarimetric imaging systems, and enhance their resistance to sensor drifts. Third, feasibility of polarimetric self-calibration can provide insights in biology: some animals have polarizationsensitive vision $[4,8,10,20,26,29,35]$. Polarimetric selfcalibration may help such animals infer their kinetic state.

\section{Acknowledgments}

Special thanks to David Diner and Michael Elad for useful discussions, Aviad Levis, Amit Aides, Mark Sheinin for giving a hand in the experimental setup, and Johanan Erez, Ina Talmon and Dani Yagodin for technical support. Thanks also to members of the Laboratory for Network Biology Research, for hosting us during an experiment. Yoav Schechner is a Landau Fellow - supported by the Taub Foundation. This research, partly supported by the Technion Funds for Security Research and the Israel Science Foundation (ISF Grant 1467/12), was conducted in the Ollendorff Minerva Center. Minerva is funded through the BMBF. 


\section{References}

[1] M. Ben-Ezra, "Segmentation with invisible keying signal," Proc. IEEE CVPR, vol. 1, pp. 32-37, 2004.

[2] I. Berezhnyy and A. Dogariu, "Time-resolved Mueller matrix imaging polarimetry ," Optics Exp. 12:4635-4649, 2004.

[3] C. Collin, S. Pattanaik, P. LiKamWa and K. Bouatouch, "Computation of polarized subsurface BRDF for rendering," Proc. Graphics Interface Conf., pp. 201-208, 2014.

[4] T. W. Cronin and J. Marshall, "Patterns and properties of polarized light in air and water," Phil. Trans. R. Soc. B 366:619-626, 2011.

[5] A. R. Dahlberg, N. J. Pust, and J. A. Shaw, "Effects of surface reflectance on skylight polarization measurements at the Mauna Loa Observatory" Optics Exp. 19:16008-16021, 2011.

[6] T. Diamant and Y. Y. Schechner, "Overcoming visual reverberations," Proc. IEEE CVPR, 2008.

[7] D. J. Diner, A. Davis, B. Hancock, S. Geier, B. Sheingans, V. Jovanovic, M. Bull, D. M. Rider, R. A. Chipman, A.-B. Mahler and S. C. McClain, "First results from a dual photoelastic-modulator-based polarimetric camera," App. Opt., vol. 49, pp. 2929-2946, 2010.

[8] C. Evangelista, P. Kraft, M. Dacke, T. Labhart and M. V. Srinivasan, "Honeybee navigation: critically examining the role of the polarization compass," Phil. Trans. R. Soc. B 369:20130037, 2014.

[9] H. Farid and E. H. Adelson, "Separating reflections from images by use of independent component analysis," JOSA A 16:2136-2145, 1999.

[10] G. Horvath and D. Varju, Polarized Light in Animal Vision: Polarization Patterns in Nature, Springer-Verlag, New York 2004.

[11] V. Gruev, J. Van der Spiegel and N. Engheta "Dual-tier thin film polymer polarization imaging sensor," Optics Exp., 18:19292-19303, 2010.

[12] G. C. Guarnera, P. Peers, P. Debevec, and A. Ghosh, "Estimating surface normals from spherical stokes reflectance fields," Proc. ECCV, LNCS 7584, pp. 340-349, 2012.

[13] M. Gupta, S. G. Narasimhan and Y. Y. Schechner, "On controlling light transport in poor visibility environments," Proc. IEEE CVPR, 2008.

[14] D. Gutierrez, S. G. Narasimhan, H. Wann Jensen, and W. Jarosz, "Scattering," Course notes, ACM Siggraph Asia Courses 2008.

[15] B. A. Hooper, B. Baxter, C. Piotrowski, J. Z. Williams and J. Dugan "An airborne imaging multispectral polarimeter," Proc. IEEE/MTS Oceans 2009.
[16] R. Horisaki, K. Choi, J. Hahn, J. Tanida and D. J. Brady, "Generalized sampling using a compound-eye imaging system for multi-dimensional object acquisition," Optics Exp., 18:19367-19378, 2010.

[17] R. Horstmeyer, G. Euliss, R. Athale and M. Levoy "Flexible multimodal camera using a light field architecture," Proc. IEEE ICCP 2009.

[18] I. Ihrke, K. N. Kutulakos, H. P. A. Lensch, M. Magnor and W. Heidrich "State of the art in transparent and specular object reconstruction,” Proc. Eurographics 2008.

[19] J. Jia and C.-K. Tang, "Image registration with global and local luminance alignment," Proc. IEEE ICCV, vol. 1, 156163, 2003.

[20] S. Johnsen, N. J. Marshall and E. A. Widder, "Polarization sensitivity as a contrast enhancer in pelagic predators: lessons from in situ polarization imaging of transparent zooplankton,” Phil. Trans. R. Soc. B 366:655-670, 2011.

[21] F. Joos, E. Buenzli, H. M. Schmid and C. Thalmann, "Reduction of polarimetric data using Mueller calculus applied to Nasmyth instruments," Proc. SPIE 7016, Observatory Operations: Strategies, Processes, and Systems II, 2008.

[22] R. Kaftory, Y. Y. Schechner and J. Zeevi, "Variational distance dependent image restoration," Proc. IEEE CVPR, 2007.

[23] G. W. Kattawar and C. N. Adams, "Stokes vector calculations of the submarine light field in an atmosphere-ocean with scattering according to a Rayleigh phase matrix: effect of interface refractive index on radiance and polarization," Limnol. Oceanogr., 34:1453-1472, 1989.

[24] N. Kong, Y.-W. Tai and J. S. Shin, "A Physically-based approach to reflection separation: from physical modeling to constrained optimization,” IEEE Trans. PAMI 36:209-221, 2014.

[25] D. Lanman, G. Wetzstein, M. Hirsch, W. Heidrich and R. Raskar, "Polarization fields: dynamic light field display using multi-layer LCDs," ACM TOG 30, Article 186, 2011.

[26] A. Lerner, S. Sabbah, C. Erlick and N. Shashar, "Navigation by light polarization in clear and turbid waters," Phil. Trans. R. Soc. B 366:671-679, 2011.

[27] A. Litvinov and Y. Y. Schechner, "Addressing radiometric nonidealities: A unified framework," Proc. IEEE CVPR, Vol. 2, pp. 52-59, 2005.

[28] A. B. Mahler, D. J. Diner, and R. A. Chipman, "Analysis of static and time-varying polarization errors in the multiangle spectropolarimetric imager," App. Opt., 50:2080-2087, 2011.

[29] L. M. Mäthger and R. T. Hanlon, "Anatomical basis for camouflaged polarized light communication in squid," Biology Letters, 2494-496, 2006. 
[30] D. Miyazaki, M. Sato, Y. Sato and K. Ikeuchi, "Determining surface orientations of transparent objects based on polarization degrees in visible and infrared wavelengths," JOSA A, vol. 19, pp. 687-694, 2002.

[31] A. Mohan, G. Woo, S. Hiura, Q. Smithwick, R. Raskar, "Bokode: imperceptible visual tags for camera based interaction from a distance," ACM TOG 28, Article 98, 2009.

[32] M. Mujat, E. Baleine, and A. Dogariu, "Interferometric imaging polarimeter," JOSA A 21:2244-2249, 1999.

[33] T. Papadhimitri and P. Favaro, "A new perspective on uncalibrated photometric stereo," Proc. IEEE CVPR, 1474-1481, 2013.

[34] D. Reddy, A. Veeraraghavan and R. Chellappa. "P2C2: Programmable pixel compressive camera for high speed imaging." Proc. IEEE CVPR, pp. 329-336, 2011.

[35] N. W. Roberts, M. L. Porter, and T. W. Cronin, "The molecular basis of mechanisms underlying polarization vision," Phil. Trans. R. Soc. B 366:627-637, 2011.

[36] Y. Y. Schechner, "Inversion by $P^{4}$ : polarization picture post-processing” Phil. Trans. R. Soc. B 366:638-648, 2011.

[37] Y. Y. Schechner, D. J. Diner and J. V. Martonchik, "Spaceborne underwater imaging," Proc. IEEE ICCP, 2011.

[38] Y. Y. Schechner and S. K. Nayar, "Generalized mosaicing: Polarization panorama,” IEEE TPAMI 27:631-636, 2005.

[39] R. Szeliski, Computer Vision: Algorithms and Applications, Chapters 6,7,10. Springer, 2011.

[40] K. Tanaka, Y. Mukaigawa, Y. Matsushita and Y. Yagi, "Descattering of transmissive observation using parallel high-frequency illumination,” Proc. IEEE ICCP 2013.

[41] J. Tinbergen, "Accurate optical polarimetry on the Nasmyth platform,” Pub. Astron. Soc. Pacific 119:1371-1384, 2007.

[42] V. Treeaporn, A. Ashok and M. A. Neifeld, "Increased field of view through optical multiplexing," Optics Exp., 18:22432-22445, 2010.

[43] T. Treibitz and Y, Y. Schechner, "Polarization: Beneficial for visibility enhancement?” Proc. IEEE CVPR, 2009.

[44] J. S. Tyo, D. L. Goldstein, D. B. Chenault and J. A. Shaw, "Review of passive imaging polarimetry for remote sensing applications," App. Opt. 45:5453-5469, 2006.

[45] F. Xu, J. E. Ford, and Y. Fainman, "Polarization-selective computer-generated holograms: design, fabrication, and applications," App. Opt., 34:256-266, 1995.

[46] S. Umeyama and G. Godin, "Separation of diffuse and specular components of surface reflection by use of polarization and statistical analysis of images," IEEE Trans. PAMI 26:639-647, 2004.
[47] L. Zhang, E. R. Hancock and G. Atiknson "Reflection component separation using statistical analysis and polarisation," Proc. PRIA, LNCS 6669, 476-483, 2011. 